\title{
Hyperhidrosis of Central Hypogonadism Leading to Diagnosis of Shapiro Syndrome: Temperature Dysregulation, Hypothermia and Agenesis of the Corpus Callosum
}

\author{
Megan A. Traxinger ${ }^{1}$, Emily A. Beckett1,2, Dana Danley1, Udaya M. Kabadi1,2* \\ ${ }^{1}$ Broadlawns Medical Center, Des Moines, USA \\ ${ }^{2}$ University of Iowa, Iowa City, USA \\ Email: mtraxinger@broadlawn s.org, ebeckett@broadlawns.org, ddanley@broadlawns.org, *ukabadi@gmail.com
}

How to cite this paper: Traxinger, M.A., Beckett, E.A., Danley, D. and Kabadi, U.M. (2019) Hyperhidrosis of Central Hypogonadism Leading to Diagnosis of Shapiro Syndrome: Temperature Dysregulation, Hypothermia and Agenesis of the Corpus Callosum. Open Journal of Endocrine and Metabolic Diseases, 9, 1-7.

https://doi.org/10.4236/ojemd.2019.91001

Received: January 3, 2019

Accepted: January 26, 2019

Published: January 29, 2019

Copyright $\odot 2019$ by author(s) and Scientific Research Publishing Inc. This work is licensed under the Creative Commons Attribution International License (CC BY 4.0).

http://creativecommons.org/licenses/by/4.0/

\begin{abstract}
Background: Central or hypothalamic hypogonadism as an initial manifestation of Shapiro Syndrome has not been described in the literature. Herein, we report first case in which initial presentation of central hypogonadism led to a confirmed diagnosis of Shapiro Syndrome during a casual evaluation of hypothalamic pituitary anatomy with MRI of brain. Case presentation: 53 year old Caucasian man was documented to manifest Central or hypogonadotropic hypogonadism following evaluation of excessive sweating episodes, lack of libido and erectile dysfunction for a duration of several years. Brain MRI performed for assessment of the etiology documented no pituitary abnormality. Instead agenesis of Corpus Callosum was noted. The subject had been hospitalized on many occasions at this and several other medical centers with hypothermia or hyperthermia without a documentation of a definite cause. Therefore, the diagnosis of Shapiro Syndrome was made. Conclusion: This report is the first documentation of subject manifesting central, more likely to be hypothalamic rather than hypogonadotropic hypogonadism in conjunction with Shapiro Syndrome.
\end{abstract}

\section{Keywords}

Hyperhidrosis, Central Hypogonadism, Temperature Dysregulation, Callosal Agenesis, Shapiro Syndrome

\section{Introduction}

Shapiro syndrome is a rare disorder characterized by the clinical triad of recur- 
rent episodes of hypothermia or fever due to temperature dysregulation, hyperhidrosis, and developmental agenesis of the corpus callosum [1] [2] [3]. Although, corpus callosal agenesis is congenital, the manifestations are not frequently recognized for several years [1]-[10]. Herein we report an adult subject who presented with hypothermia and hyperhidrosis. On further inquiry, the subject reported progressive decline in libido and erectile function as well as fatigue and muscle weakness for the duration of over 20 years. Appropriate evaluation confirmed the diagnosis of central hypogonadism, either of hypothalamic or hypogonadotropic etiology and therefore, hyperhidrosis was initially attributed to hypogonadism as well. MRI of brain was performed to evaluate anatomy of hypothalamus and pituitary gland because of presence of central hypogonadism to define etiology and recommend appropriately therapeutic measures. MRI documented agenesis of corpus callosum and thus established the diagnosis of Shapiro Syndrome. We believe that all these manifestations including hypogonadism may be induced by hypothalamic dysfunction implicated in pathophysiology of Shapiro Syndrome. Therefore, our intent to report this subject is to add central hypogonadism of hypothalamic etiology as one of the manifestations of Shapiro syndrome and may be partially responsible for hyperhidrosis.

\section{Case Presentation}

53 year old Caucasian male presented to the emergency department (ED) with complaints of generalized weakness, vague abdominal discomfort, nausea, hyperhidrosis and shaking chills. On further inquiry, subject reported several hospitalizations for similar symptoms, occasionally with fever of unknown origin during past 25 years. The episodes had occurred prominently during summers following exposure to hot humid sunny days while working outdoors. During some of these events, subject reported experiencing headache, vomiting and disorientation though without any other neurological symptoms including seizure, visual problems or imbalance. Treatments with hydroxyzine, diazepam, and carbamazepine were unsuccessful at alleviating symptoms. Subject denied smoking, alcohol ingestion or use of illicit drugs including marijuana and over the counter supplements. Past medical history included hypertension, depression and anxiety treated with daily administration of lisinopril, $10 \mathrm{mg}$. and bupropion, $150 \mathrm{mg}$. and lorazepam, $1 \mathrm{mg}$. as needed respectively as well as nephrolithiasis. Surgical history revealed 2 exploratory leparotomies during infancy to relieve small bowel obstruction caused by mesenteric bands and adhesions. Prophylactic appendectomy was performed during one of the procedures. Family history was non contributory.

Physical examination showed alert, oriented man looking appropriate for his stated age and in no acute distress. Vital signs revealed hypothermia with a rectal temperature, 94.7 degree $\mathrm{F}$, pulse $94 / \mathrm{min}$, respiratory rate $16 / \mathrm{min}$ and blood pressure, 124/72 mm Hg. without orthostasis. HEENT examination was unremarkable including intact visual fields bilaterally. Examination of neck showed a 
multinodular goiter with a freely mobile prominent firm nodule about 1.2 CM in diameter without a bruit in the right lobe. No cervical lymphadenopathy was appreciated. Heart sounds were normal and without a murmur. Lungs were clear to auscultation with normal breath sounds devoid of adventitious sounds. Abdomen was soft, non tender and without organomegaly, ascites or bruits. Bowel sounds appeared hypoactive. Neurological examination was intact with no focal deficit. Diffuse, blanching erythema was evident on upper extremities. However, peripheral pulses in both upper and lower extremities were well palpated and there was no edema. No clubbing or cyanosis of fingers or toes was present. Testicular examination was normal and gynecomastia was absent.

Extensive laboratory testing showed normal complete blood count, erythrocyte sedimentation rate, C-reactive protein, INR and partial thromboplastin time. Serum chemistries including creatinine, urea nitrogen, liver enzymes, lipid panel as well as free T4 and TSH were normal. Urinalysis was normal as well. Other ancillary evaluation including tilt table testing, thermoregulatory sweat testing, autonomic reflex screening was unremarkable. Subject was discharged after 2 days of observation following resolution of symptoms without establishing the definitive etiology of symptomatology. Outpatient endocrinology consultation was also sought for assessment of symptoms prior to discharge and subject was requested to return to Outpatient clinic in 4 weeks for further evaluation.

As per endocrinology consultation, serum concentrations of serotonin, cortisol and fractionated metanephrines were determined to be within normal ranges. However, both serum Free (2.2 ng/dl, normal range; 4.5 - 17.2) and total testosterone (155 ng/dl, normal range; 300 - 1000) were markedly decreased while LH was subnormal but with normal FSH and prolactin levels. 24 hour urinary excretions of 5 HIAA, catecholamines and free cortisol were normal. Thyroid ultrasound examination confirmed the presence of hypoechoic nodule in the right lobe and the biopsy showed a benign colloid nature. Diagnosis of central hypogonadism was confirmed by repeated testing at about 2 weeks after discharge. Evaluation for etiology of central hypogonadism revealed markedly elevated serum ferritin concentration $>1000 \mathrm{ng} / \mathrm{dl}$ on more than one occasion. MRI of the brain to assess the cause of central hypogonadism showed no abnormality of the pituitary gland and agenesis of corpus callosum (Figure 1). Bone mineral density assessed because of presence of hypogonadism showed osteopenia. At this juncture, therapy with topical testosterone gel $5 \mathrm{gm}$ daily, oral alendronate $70 \mathrm{mg}$ weekly and phlebotomy were initiated for management of central hypogonadism, osteopenia and hemochromatosis respectively. L thyroxine was also prescribed to suppress further enlargement of the documented nodules and prevent formation of new ones. During follow up visits at 3 and 6 months, subject reported improvement in fatigue, lethargy, libido and erectile function as well as remission of hyperhydrosis and without requirement for hospitalization but also without a total resolution of hypothermia. Oral administration of clonidine, $0.1 \mathrm{mg}$ at bedtime initiated because of its previously 

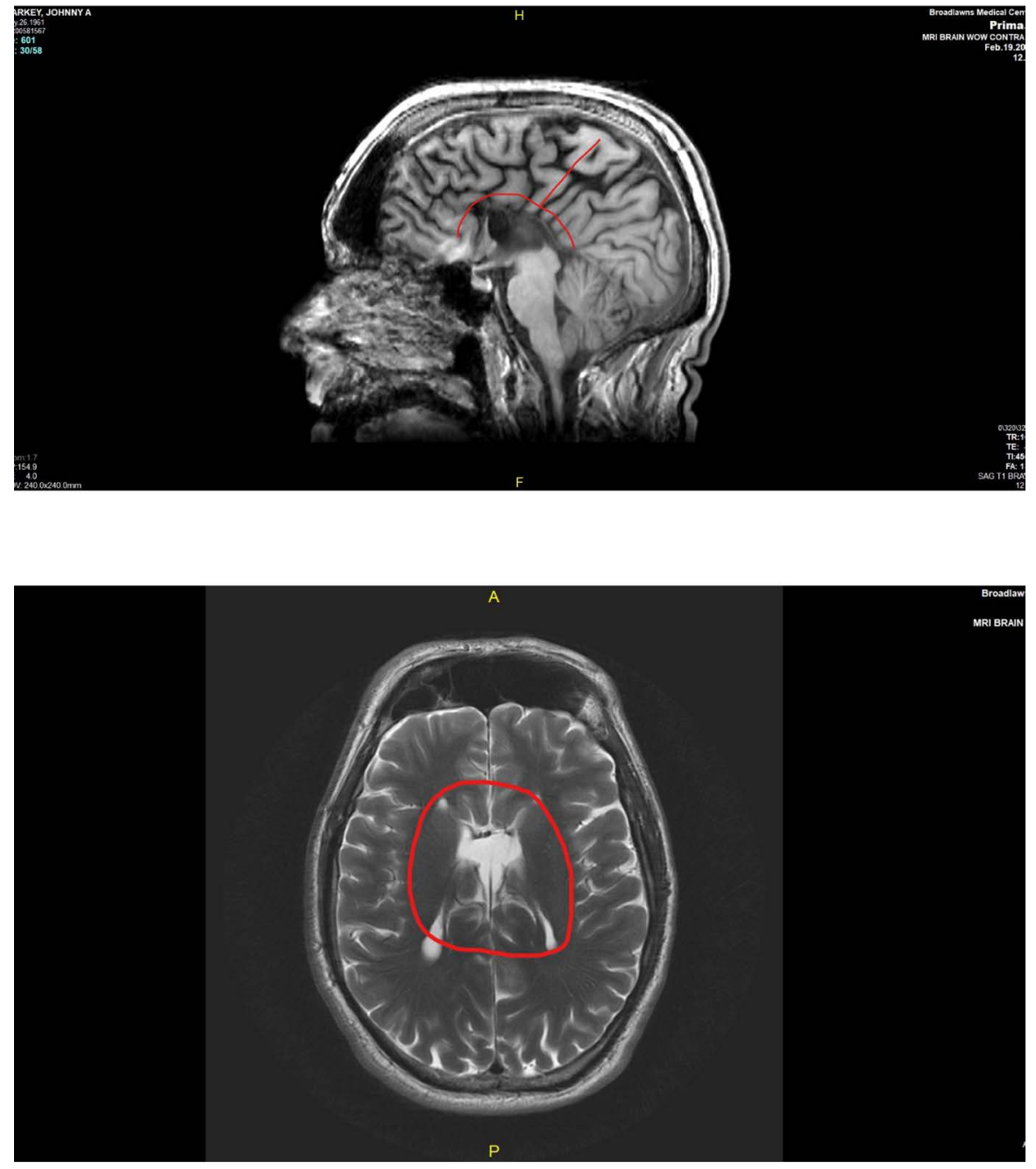

Figure 1. MRI of Brain: lateral and anteroposterior views showing Absent Corpus Callosum.

documented efficacy in remission of symptoms resulted in further improvement in all manifestations [3] [4] [5].

\section{Discussion}

Shapiro syndrome is a rare disorder characterized by the clinical triad of recurrent episodic hypothermia, hyperhidrosis, and developmental agenesis of the corpus callosum [1] [2] [3]. It was first described in 1969 by Shapiro and Plum (1) To date, less than 60 cases have been reported in the literature [1]-[15]. Age of onset has ranged from six months to 80 years $(14,15)$. However, the diagnosis is delayed for several years in majority of subjects because of nonspecific manifestations [1]-[6] [14] [15].

Agenesis of the corpus callosum is a hallmark of Shapiro syndrome. However, all the manifestations can not be attributed to this congenital anomaly alone since it is a relatively common asymptomatic isolated developmental defect without specific clinical manifestations in majority of subjects [1] [14] [15]. Moreo- 
ver, Shapiro syndrome has not been documented to ensue following elective surgical callosotomy. Finally, abnormalities of the corpus callosum may occur as isolated defects or may be associated with other brain malformations. Therefore, clinical manifestations are varied in terms of the degree of severity and multiplicity depending on accompanying structural anomalies [1] [16] [17]. Alternatively, many familial cases with a Shapiro phenotype with presence of normal callosal structure have been described [14]. Therefore, other causative factors are likely to contribute to these clinical manifestations.

Several other possible causal mechanisms have been proposed. Thermal dysregulation, especially hypothermia is postulated to result from changes in the set point of the hypothalamic thermostat, a distinct hypothalamic dysfunction attributed to probable structural abnormalities, e.g. degenerative changes, neurochemical dysfunction, inflammatory processes, other irritative mechanisms such as autoimmune disease or immunologic processes, and seizure activity etc [3] [8] [11] [15] [16].We believe that hyperhydrosis was primarily induced by central hypogonadism in our subject since almost total remission ensued following topical testosterone therapy. This manifestation is similar to hypothalamic hypothyroidism documented in a case report [11]. It is apparent that central hypogonadism is not included as a manifestation of Shapiro Syndrome most likely because of lack of appropriate complete evaluation of hypogonadism, a well established contributor to hyperhydrosis. Finally, multinodular goiter noted in our subject appears to be an innocent association as it is a frequent finding in a general population.

Therapies with administration of numerous drugs to alleviate manifestations of Shapiro syndrome have been attempted, although with varying degrees of success. Efficacy of such treatments is difficult to assess due to a multiple factors including limited data in the literature other than a few case reports because of the rare occurrence of the disorder, lack of recognition due to absence of classical clinical manifestations, periodic nature of disease and the absence of an animal model (4). Pharmacologic approaches have been of empirical nature at best and have included dopamine antagonist chlorpromazine, serotonin antagonist cyprohepatidine, and $\alpha_{2}$-adrenergic agonists including clonidine all with inconsistent results [3] [4] [5] [15] [16]. Limited data supports use of clonidine in preference to other drugs. Clonidine has been proposed to act by regulating the hypothalamic dysfunction, indicating that the primary defect may relate to inadequate adrenergic activity in the hypothalamus [3] [4] [5] [7]. Several case reports have documented partial or complete remission at three months after initiation of clonidine $0.1 \mathrm{mg}$ orally twice daily [3] [4] [5]. Therefore, clonidine was initiated while continuing therapy with testosterone and levothyroxine which appeared to have resulted in remission of all symptoms, e.g. hyperhydrosis, temperature regulation, decreased lipids, erectile dysfunction, fatigue etc. for 2 years.

\section{Conclusion}

Herein, we report probably the first patient presenting with hypogonadotropic 
hypogonadism as an initial manifestation leading to diagnosis of Shapiro Syndrome following MRI evaluation of Hypothalamic Pituitary anatomy showing agenesis of Corpus Callosum. Therefore, all the manifestations of Shapiro Syndrome including hypogonadism may be attributed to hypothalamic dysfunction.

\section{Acknowledgements}

The authors are indebted to the subject for providing informed consent for publication of this report.

\section{Conflicts of Interest}

The authors declare no conflicts of interest regarding the publication of this paper.

\section{References}

[1] Shapiro, W.R., Williams, G.H. and Plum, F. (1969) Spontaneous Recurrent Hypothermia Accompanying Agenesis of the Corpus Callosum. Brain, 92, 423-436. https://doi.org/10.1093/brain/92.2.423

[2] Kenney, D.L., Toledano, M. and Moseley, B.D. (2012) Teaching NeuroImages: Hypothermia and Corpus Callosum Agenesis in Shapiro Syndrome: Too Cold, Even for a Viking. Neurology, 79, e78. https://doi.org/10.1212/WNL.0b013e318266fc66

[3] Shenoy, C. (2008) Shapiro Syndrome. QJM: An International Journal of Medicine, 101, 61-62. https://doi.org/10.1093/qjmed/hcm106

[4] Darku, B., Kalra, P., Prasad, C. and Yadav, R. (2011) Episodic Hyperhydrosis with Corpus Callosum Agenesis: A Rare Case of Shapiro Syndrome. Neurology India, 59, 130-131. https://doi.org/10.4103/0028-3886.76868

[5] Dundar, N.O., Boz, A., Duman, O., Aydin, F. and Haspolat, S. (2008) Spontaneous Periodic Hypothermia and Hyperhydrosis. Pediatric Neurology, 39, 438-440. https://doi.org/10.1016/j.pediatrneurol.2008.08.010

[6] Walker, B.R., Anderson, J. and Edwards, C.R.W. (1992) Clonidine Therapy for Shapiro's Syndrome. Quarterly Journal of Medicine, 82, 235-245.

[7] Harden, P.N., Bone, I. and Rodger, R.S.C. (1994) Acute Renal Failure Due to Shapiro's Syndrome. Nephrology Dialysis Transplantation, 9, 426-427.

[8] Arkader, R. and Takeuchi, C.A. (2008) Shapiro Syndrome with Hypothalamic Hypothyroidism. Arquivos de Neuro-Psiquiatria, 66, 418-419. https://doi.org/10.1590/S0004-282X2008000300029

[9] Tambasco, N., Corea, F. and Bocola, V. (2005) Subtotal Corpus Callosum Agenesis with Recurrent Hyperhydrosis-Hypothermia (Shapiro Syndrome). Neurology, 65, 124. https://doi.org/10.1212/01.WNL.0000160423.17825.5E

[10] Owen, A.J. and Backman, S.B. (2001) Perioperative Temperature Changes in a Patient with Shapiro Syndrome. Anesthesiology, 95, 268-270. https://doi.org/10.1097/00000542-200107000-00042

[11] Belcastro, V., Striano, P., Pierguidi, L., Arnaboldi, M. and Tambasco, N. (2012) Recurrent Hypothermia with Hyperhidrosis in Two Siblings: Familial Shapiro Syndrome Variant. Journal of Neurology, 259, 756-758. https://doi.org/10.1007/s00415-011-6237-0

[12] Topcu, Y., Bavram, E., Karaoglu, P., Yis, U. and Kurul, S.H. (2013) The Combina- 
tion of Thermal Dysregulation and Agenesis of Corpus Callosum: Shapiro's or/and Reverse Shapiro's Syndrome. Annals of Indian Academy of Neurology, 16, 716-719. https://doi.org/10.4103/0972-2327.120451

[13] Dutau, G., Carriere, J.P. and Rochiccioli, P. (1975) Shapiro's Syndrome. Recurrent Spontaneous Hypothermia with Hypoplasia of the Corpus Callosum. Pediatrie, 30, 117-127.

[14] Pazkerska, A., O’Connell, M., Pender, N., Gavin, C., Murray, B. and O’Dowd, S. (2013) Insights into Thermoregulation: A Clinic-Radiological Description of Shapiro Syndrome. Journal of the Neurological Sciences, 329, 66-68. https://doi.org/10.1016/j.jns.2013.03.014

[15] Barkovich, A.J., Simon, E.M. and Walsh, C.A. (2001) Callosal agenesis with Cyst: A Better Understanding and New Classification. Neurology, 56, 220-227. https://doi.org/10.1212/WNL.56.2.220

[16] Bedeschi, M.F., Bonaglia, M.C., Grasso, R., Pellegri, A., Garghentino, R.R., Battaglia, M.A., et al. (2006) Agenesis of the Corpus Callosum: Clinical and Genetic Study in 63 Young Patients. Pediatric Neurology, 34, 186-193. https://doi.org/10.1016/j.pediatrneurol.2005.08.008

[17] Kloos, R.T. (1995) Spontaneous Periodic Hypothermia. Medicine, 74, 268-280. https://doi.org/10.1097/00005792-199509000-00004 